\title{
Feasibility of Adjustable Electrodes for Radiofrequency Ablation of Benign Thyroid Nodules
}

Jiyeong Lee, MD, Jung Hee Shin, MD, PhD, Soo Yeon Hahn, MD, PhD, Ko Woon Park, MD, Ji Soo Choi, MD, PhD

All authors: Department of Radiology, Samsung Medical Center, Sungkyunkwan University School of Medicine, Seoul, Korea

Objective: The purpose of this study was to evaluate a novel radiofrequency ablation (RFA) application utilizing an adjustable electrode for treatment of benign thyroid nodules.

Materials and Methods: This study included 21 patients who had undergone RFA treatment on 21 thyroid nodules, utilizing an 18-gauge internally cooled electrode equipped with a size adjustable active tip. The peripheral nodule portions were ablated with the moving-shot technique and a shorter active tip, and the nodule centers were ablated with the fixed technique and a longer active tip. We assessed parameters including characteristics of the treated nodules, use of variable-sized active tips, volume reduction rate, therapeutic success rate, and post-procedural complications. The therapeutic success rate was defined as a $>50 \%$ volume reduction of the initial nodule volume at the 6 - or 12-month follow-up.

Results: The treated thyroid nodules were large enough to cause symptoms (mean volume, $29.6 \mathrm{~mL}$ ). Two types of active tips per session were used for all nodules. The mean volume reduction rate at the last follow-up was $68.3 \pm 4.4 \%$ and our therapeutic success rate was $90.5 \%$. Both symptoms and cosmetic scores decreased significantly. Minor complications in three patients were recorded during and after the procedure.

Conclusion: This initial study demonstrated that an adjustable electrode for RFA of benign thyroid nodules effectively and safely resulted in volume reduction.

Keywords: Thyroid nodule; Ultrasonography; Safety; Radiofrequency ablation

\section{INTRODUCTION}

Thyroid nodules are a common clinical problem, and their incidence has increased with the recent application of thyroid ultrasound (US) $(1,2)$. US-guided radiofrequency ablation (RFA) for thyroid nodules has been accepted as a safe and effective treatment modality (3-5), and largely replaced surgery for the treatment of symptomatic benign

Received September 30, 2019; accepted after revision December 29, 2019.

Corresponding author: Jung Hee Shin MD, PhD, Department of Radiology, Samsung Medical Center, Sungkyunkwan University School of Medicine, 81 Irwon-ro, Gangnam-gu, Seoul 06351, Korea.

- Tel: (822) 3410-2548 • Fax: (822) 3410-0049

- E-mail: jhshin11@skku.edu

This is an Open Access article distributed under the terms of the Creative Commons Attribution Non-Commercial License (https://creativecommons.org/licenses/by-nc/4.0) which permits unrestricted non-commercial use, distribution, and reproduction in any medium, provided the original work is properly cited. thyroid lesions. RFA results in significant improvement of thyroid nodule-related symptoms and cosmetic problems by reducing the nodule volume $(6,7)$.

Unlike hepatic tumors, thyroid nodules are relatively elliptical, and do not have sufficient boundaries separating them from the surrounding major structures. Thus, a designated electrode for thyroid nodules (different from the conventional electrode used for RFA of liver tumors) is needed. The modified straight electrodes designed for thyroid nodule ablation are short $(7 \mathrm{~cm})$, making them easy to control, and thin (18-gauge), thereby minimizing injury to the surrounding major structures. An electrode with an active tip of a specific length (for example, $0.5,0.7,1.0$, or $1.5 \mathrm{~cm}$ ) should be chosen according to the tumor size (8). Therefore, small-sized active tips may be used effectively for small-sized thyroid nodules (9). However, commercially available RF electrodes only have a fixed-sized active tip appropriate for a single procedure.

There are situations in clinical practice when the use 
of more than one size-type RF electrode per session is necessary, such as when peripheral small units should be treated with a longer tip, and when higher power is required to ablate a large mass with a smaller tip. Due to the medical expense, it is not cost effective to use multiple RF electrodes for one procedure. Consequently, we decided to utilize a novel RF electrode equipped with an adjustable active tip (initially designed for hepatic tumors), but with a diameter equivalent to conventional non-adjustable electrodes designed for thyroid nodules (10). Additionally, we did not initially modify the adjustable $10 \mathrm{~cm}$ total length electrode specifically for thyroid nodule ablation. Subsequently, the total electrode length was reduced to 8 $\mathrm{cm}$ equivalent to a dedicated thyroid electrode. To the best of our knowledge, there have been no published studies demonstrating the usefulness of RFA for thyroid nodules using an adjustable active tip in a single internally cooled electrode. Therefore, the purpose of this study was to evaluate a novel RFA application utilizing an adjustable electrode for treatment of benign thyroid nodules.

\section{MATERIALS AND METHODS}

\section{Patients}

Our local institutional review board approved this retrospective study (2019-06-073-001), and waived the requirement for informed consent. Twenty-nine patients underwent RFA treatment of thyroid nodules with 18-gauge internally cooled adjustable electrodes, at our institution between April 2011 and December 2018. Following treatment, seven patients did not continue the follow-up protocol. One patient had a subcentimeter-sized nodule, but insisted on receiving treatment due to nodule phobia. Finally, 21 nodules from 21 patients were included in the present study. We made an empirical decision to use adjustable electrodes for nodules that measured more than $3 \mathrm{~cm}$ in the longest diameter. The inclusion criteria were as follows, according to the Korean thyroid RFA guidelines (11): 1) nodules measuring $3 \mathrm{~cm}$ or more in diameter, 2) symptomatic or cosmetic problems due to thyroid nodules, 3) benign thyroid nodules diagnosed at least twice with cytology, or diagnosed with fine-needle aspiration or coreneedle biopsy, and retaining highly specific, benign US features (i.e., isoechoic spongiform nodules, or partially cystic nodules with an intracystic comet tail artifact), 4 ) solid ( $\leq 10 \%$ fluid component) or predominantly solid (11-50\% fluid component) thyroid nodules, and 5) serum thyroid hormone and thyrotropin levels within normal range $(11,12)$. The exclusion criteria were as follows: 1) a followup period of less than 6 months and 2) cysts or cystic nodules (> 90\% fluid component) (13).

\section{Pre-Procedural Evaluation}

All patients underwent US examinations, US-guided aspiration or biopsy, a thyroid function test, a bleeding tendency test, and a clinical examination. Thyroid US examinations were conducted with a 5-12 MHz linear transducer (iU22, Philips Healthcare, Seattle, WA, USA). Each nodule was measured, and the three orthogonal diameters (the largest and the other two diameters perpendicular to it) were used to calculate the volume with the equation $V=\pi a b c / 6$ (where $V$ is volume, $a$ is the largest diameter, and $b$ and $c$ are the two perpendicular diameters). In addition, the solid component portion of the nodule was evaluated with US. All patients were asked to rate their nodule-related symptom score on a $10-\mathrm{cm}$ visual analogue scale (0-10) before the RFA procedure, and the cosmetic score was recorded by the physician (1, no palpable mass; 2 , a palpable mass without cosmetic problems; 3, cosmetic problems only when swallowing; and 4 , readily detected cosmetic problems) (5).

\section{Procedures}

RFA was conducted on an outpatient basis under US guidance by a thyroid radiologist with 10 years of experience in thyroid RFA. An 18-gauge internally cooled adjustable RF electrode with a variable-sized active tip, and a total length of 10 or $8 \mathrm{~cm}$ (Proteus RF electrodes, STARmed, Goyang, Korea) was used in the procedures. The adjustable electrode diameter was equivalent to conventional non-adjustable electrodes designed for thyroid nodules. The exposed (non-insulated) distal tip acted as an active segment for RF energy discharge, and the length of the exposed active tip could be easily adjusted from $5 \mathrm{~mm}$ to $30 \mathrm{~mm}$ (in $5 \mathrm{~mm}$ step variables) by simply sliding the button on the handle (Fig. 1).

Patients were instructed to lie on the bed in a supine position with a mild neck extension. After skin sterilization, local anesthetic (1-2\% lidocaine) was typically injected around the skin puncture site. The fundamental method for thyroid RFA (5) is a trans-isthmic approach involving insertion of the RF electrode through the isthmus to the target nodule, thus preventing a change in the electrode position and avoiding damage to the critical surrounding 
structures. With this approach, the operator may continuously monitor the relative position of the target nodule and electrode tip to the recurrent laryngeal nerve, thereby minimizing the risk of thermal injury during the procedure.

Another key method for RFA of thyroid nodules is the moving-shot technique (14). Unlike hepatic tumors, thyroid nodules are often ellipsoidal and exophytic from the thyroid gland, making them difficult to ablate using the fixed technique. Therefore, it is advantageous to treat a thyroid nodule by dividing it into multiple small ablation units and then treating each ablation unit separately $(14,15)$. To avoid visual disturbances caused by echogenic air bubbles, the electrode tip can initially be placed in the deepest and most remote portion of the nodule, and then pulled backward to the most superficial and accessible portion. In our study, the target nodules were divided into multiple predicted ablation units before the procedure. We created smaller units in the periphery of the target nodule and close to critical neck structures, and larger units in the central portion of the nodule (Fig. 2). Initially, by controlling the button in a single electrode, the smaller peripheral units were treated with the moving-shot technique utilizing the shorter active tip of the adjustable electrode, and subsequently the larger central units were treated with the fixed technique utilizing the longer active tip. The RF

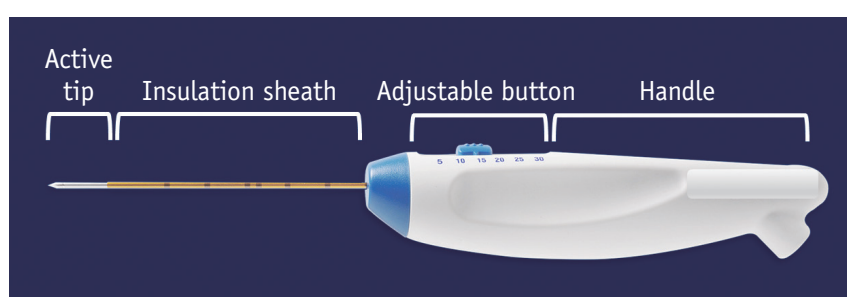

Fig. 1. Photograph of adjustable RF electrode (18 gauge, total length $8 \mathrm{~cm}$ ). Simply sliding button enables operator to control length of exposed active tip. $\mathrm{RF}=$ radiofrequency

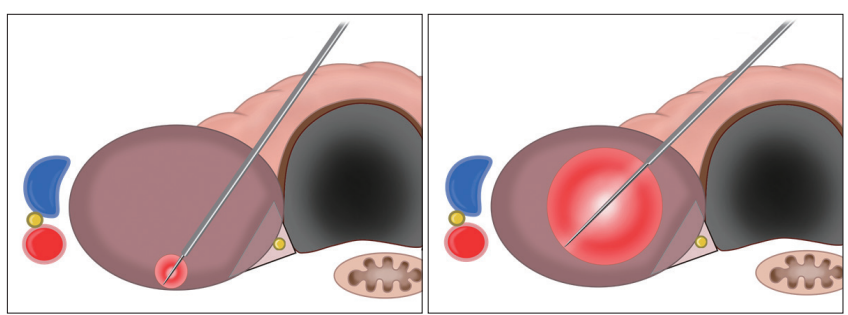

Fig. 2. Schema of adjustable electrode procedure. Electrode is inserted by trans-isthmic approach. Smaller active tip (right) may be applied to smaller units in periphery of nodule, and larger active tip (left) may be used to ablate larger units in central portion of nodule, thereby completing RFA procedure with single electrode. RFA = radiofrequency ablation power may be increased as needed when using the longer tips, however if the procedure became too painful, the power could be reduced or shut off for several seconds, or alternatively additional lidocaine could be injected around the thyroid. The ablation procedure was terminated when the target nodule became entirely transient hyperechoic. We evaluated the variable-sized active tip lengths of the adjustable electrode, procedure duration, and intra- or immediate post-procedural complications in all patients.

\section{Follow-Up}

Follow-up US examinations were usually performed at 1-6 months after RFA, and annually thereafter. Similar to the evaluations performed before ablation, the thyroid nodule volume, largest diameter, and symptom and cosmetic scores were assessed. The volume reduction rate was estimated from a single session, and was obtained as follows: volume reduction rate $=([$ initial volume - final volume $] \times 100) /$ initial volume (16). Therapeutic success was defined as $a>50 \%$ volume reduction of the initial nodule volume measured at the 12-month follow-up US examination, or at 6 months (if the follow-up period was less than 12 months) (17). The therapeutic success rate was defined as the percentage of successfully treated nodules (12). Additional treatment was allowed if the nodule US performed during the follow-up visit revealed a remaining viable portion with a potential to regrow, or if the patient complained of insufficient resolution of the symptomatic or cosmetic problems.

\section{Statistical Analysis}

The normal distribution values of the largest diameters of the nodules pre- and post-RFA were compared using the paired $t$ test. The outcomes, including volume changes, and symptom and cosmetic scores before and after RFA, were evaluated using the Wilcoxon signed-rank test. A $p$ value of $<0.05$ was considered statistically significant for all statistical analyses performed with IBM SPSS software, version 22 (IBM Corp., Armonk, NY, USA). Continuous variables are reported as the mean \pm standard deviation along with the range.

\section{RESULTS}

The mean follow-up period was 18.2 months (range, 6-71 months). Twelve patients were followed-up for 6 months, eight patients at 6,12 months and annually thereafter, 
and one patient only after 12 months. The patient characteristics are summarized in Table 1. Before RFA, the thyroid nodules were large enough to cause symptoms, with a mean volume of $29.6 \pm 6.3 \mathrm{~mL}$ (range, 3.3-110.6 mL). Solid and predominantly solid nodules were found in 18 and 3 patients, respectively. The treatment characteristics of the thyroid nodules included in the study are summarized in Table 2. The mean RF power was $72.9 \pm 23.1 \mathrm{~W}$ (range, 30-125 W), and the mean active ablation time was 22.0 \pm 8.9 minutes (range, $9-36$ minutes). The mean total amount of applied energy was $95172.3 \pm 56533.3 \mathrm{~J}$ (range, $45360-247500 \mathrm{~J}$ ). The active tip lengths varied from 0.5 $\mathrm{cm}$ to $2.0 \mathrm{~cm}$. We used two types of active tips (specifically, $0.5 \mathrm{~cm} / 1.0 \mathrm{~cm}$ in 15 patients, $0.5 \mathrm{~cm} / 1.5 \mathrm{~cm}$ in 1 patient, $1 \mathrm{~cm} / 1.5 \mathrm{~cm}$ in 2 patients, and $1.5 \mathrm{~cm} / 2 \mathrm{~cm}$ in 3 patients) for all nodules. Switching from a short active tip to a longer active tip allowed a 20-30 watt increase in applied RF power.

Treatment outcomes after RFA with adjustable electrodes are summarized in Table 3. The mean volume reduction rate at 6 or 12 months was $68.3 \pm 4.4 \%$ (range, $29.7-96.8 \%$ ), and the therapeutic success rate was $90.5 \%$ (19/21). Both the symptom and cosmetic scores decreased significantly, and most nodules (19/21) were subjected to only one session. Two therapeutic failure patients were followedup 6 months after the procedure, and after additional

Table 1. Characteristics of Study Population

\begin{tabular}{lc}
\hline \multicolumn{1}{c}{ Characteristics } & Overall $(\mathrm{n}=21)$ \\
\hline Sex (female:male) & $18: 3$ \\
Mean age (year) & $40.5 \pm 2.6(25-76)$ \\
Nodule diameter $(\mathrm{cm})$ & $4.8 \pm 0.3(3.0-7.8)$ \\
Nodule volume $(\mathrm{mL})$ & $29.6 \pm 6.3(3.3-110.6)$ \\
Symptom score & $5.4 \pm 0.4(2-8)$ \\
Cosmetic score & $3.8 \pm 0.1(1-4)$ \\
\hline
\end{tabular}

Values are presented as mean \pm standard deviation; numbers in parentheses represent range.

Table 2. Heat and Time Used for RFA of Benign Thyroid Nodules

\begin{tabular}{lc}
\hline \multicolumn{1}{c}{ Variables } & RFA with an Adjustable Electrode \\
\hline RF power $(\mathrm{W})$ & $72.9 \pm 23.1(30-125)$ \\
Ablation time $(\mathrm{min})$ & $22.0 \pm 8.9(9-36)$ \\
Total energy $(\mathrm{J})$ & $95172.3 \pm 56533.3(45360-247500)$ \\
$\begin{array}{l}\text { Delivered energy/ } \\
\text { tumor volume }(\mathrm{J} / \mathrm{mL})\end{array}$ & $6088.5 \pm 4777.4(922.5-16266.6)$ \\
\hline
\end{tabular}

Values represent mean \pm standard deviation; numbers in parenthesis represent range. Total amount of energy applied was calculated by multiplying RF power as watts and ablation time in seconds. RFA = radiofrequency ablation
RFA treatments with conventional electrodes at 7 and 10 months, respectively, the nodules were further reduced in size (Fig. 3). A fixed conventional electrode was used in the additional ablations since the tumors were not as large as in the initial procedure. No major complications were reported. However, minor complications occurred (hematoma in two patients and a skin burn in one patient), with an overall rate of $14.3 \%(3 / 21)$. The hematomas were resolved with manual compression. The second-degree skin burn occurred during the treatment of an isthmic nodule located directly beneath the epidermis in a patient who underwent a previous lobectomy; however, after topical application of an ointment to the affected area, the burn was healed.

\section{DISCUSSION}

Our study demonstrated that RFA of benign thyroid nodules using an adjustable electrode initially designed for hepatic tumors is technically feasible, efficacious, and safe. When initially introduced, the adjustable electrode was used for thyroid nodules of all sizes; however, our experience was that the adjustable electrode was more effective and valuable for larger nodules. In fact, when the adjustable electrode was used for sub-centimeter sized nodules, it was equivalent to fixed type electrodes.

Since the modified straight electrodes were developed for relatively large thyroid nodules with elliptical shapes and narrow safety margins (in contrast to hepatic tumors), a number of studies have evaluated the efficacy and safety of RFA treatment for benign thyroid nodules $(6,17,18)$. Notably, there is a requirement for more than one sizetype of electrode during the RFA procedure. Based on previous reports, the trans-isthmic approach and movingshot technique $(5,14,15)$ are two fundamental methods

Table 3. Outcomes after RFA with Adjustable Electrodes

\begin{tabular}{lccc}
\hline \multicolumn{1}{c}{ Variables } & Pre RFA & Post RFA & $P$ \\
\hline Largest diameter $(\mathrm{cm})$ & $4.8 \pm 0.3$ & $3.1 \pm 0.3$ & $<0.001^{\ddagger}$ \\
Volume $(\mathrm{mL})$ & $29.6 \pm 6.3$ & $12.0 \pm 3.8$ & $<0.001^{\S}$ \\
Volume reduction rate & & $68.3 \pm 4.4 \%$ & \\
Therapeutic success & & & $90.5 \%(19 / 21)$ \\
Symptom score & $5.4 \pm 0.4$ & $2.4 \pm 0.3$ & $<0.001^{\S}$ \\
Cosmetic score & $3.8 \pm 0.1$ & $8.6 \pm 0.7$ & $<0.001^{\S}$ \\
\hline
\end{tabular}

Values are presented as mean \pm standard deviation. * Entire data of post RFA was calculated at 12-month follow-up or 6-month followup when follow-up period was less than 12 months, ${ }^{\dagger}$ Therapeutic success (volume reduction $>50 \%$ ) at 12 -month follow-up or 6-month follow-up when follow-up period was less than 12 months, ${ }^{\ddagger}$ paired $t$ test, ${ }^{\S}$ Wilcoxon signed rank test. 

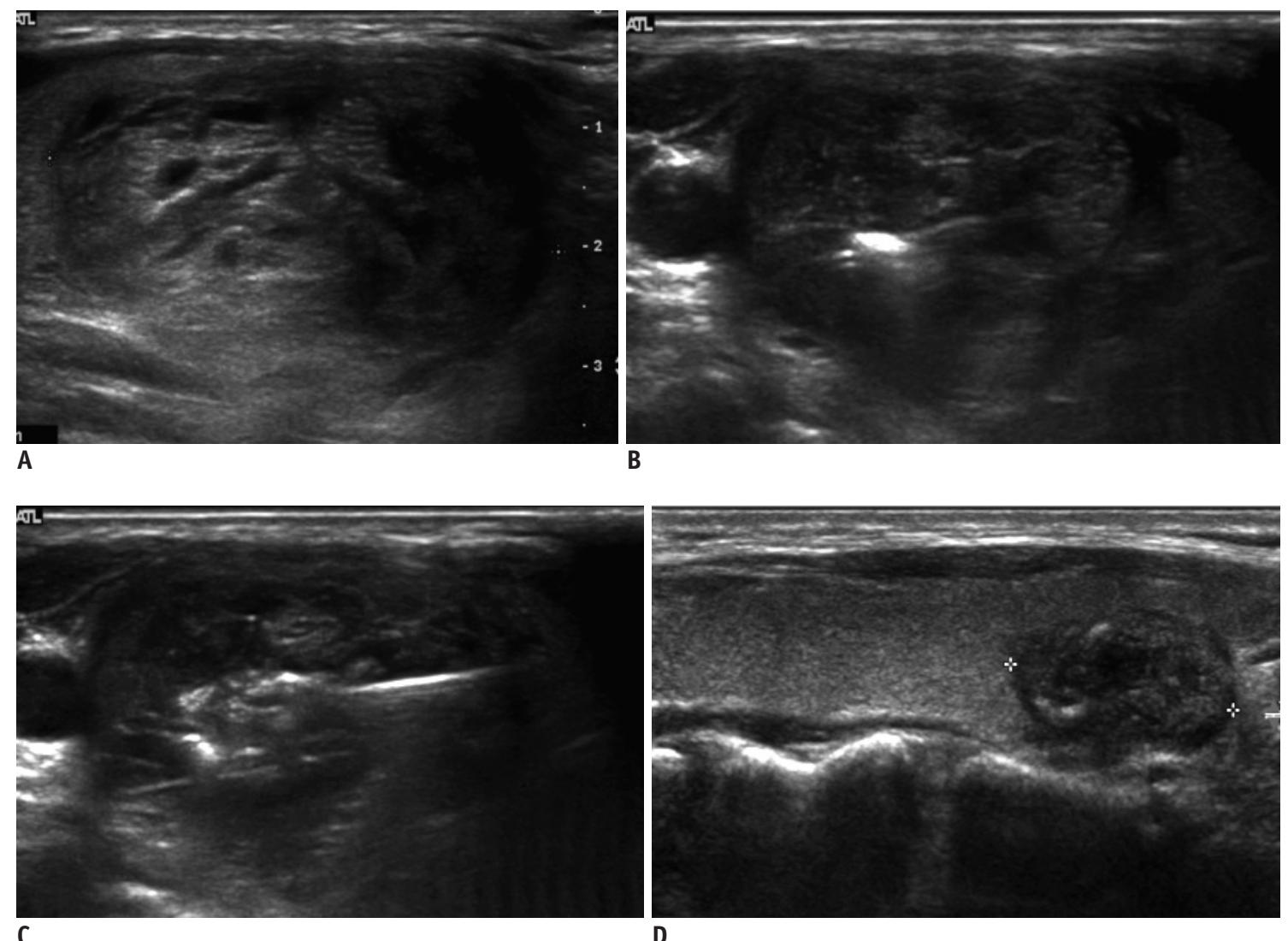

Fig. 3. RFA using adjustable electrode for benign thyroid nodule.

A. 33-year-old woman with thyroid nodule measuring $4.4 \mathrm{~cm}$ across largest diameter and $25.5 \mathrm{~mL}$ volume. Patient symptom and cosmetic scores were both 4. 1-cm active tip was applied to peripheral portion (B) and 1.5-cm active tip was applied to central portion (C) for treatment of this large-sized nodule. Since nodule was too large to treat in single session, medial section was left untreated (not shown). Additional ablation with fixed electrode was performed for remnant lesion. Six years later, follow-up ultrasound demonstrates that nodule (crosses) (D) has been reduced to $1 \mathrm{~mL}$ (volume reduction rate: $96.1 \%$ ), and symptom and cosmetic scores were 0 and 1 , respectively.

for thyroid RFA treatment. When we applied the modified moving-shot technique via the trans-isthmic approach, we used a single adjustable electrode with a short active tip for the smaller tumor units, and a longer active tip for the larger units. Our assumption was that this new type of adjustable electrode could lead to an effective tumor volume reduction, while reducing the procedural time without increasing the rate of post-procedural complications.

In 2015, a meta-analysis (19) reported that the pooled percentage mean change in nodule volume at the 6 -month follow-up was $76.1 \%$, including single and multi-sessions. Jung et al. (17) reported the efficacy and safety of RFA for 276 benign thyroid nodules in a prospective multicenter study. The mean number of RF sessions was 1.3 , the mean volume reduction rate at 12 months was $80.2 \%$, and the therapeutic success was $97.8 \%$. In previous studies, Jeong et al. (6) and Spiezia et al. (7) reported a volume reduction rate of $84.1 \%$ (at the last follow-up) and $79.4 \%$ (at 12 months), respectively. Similarly, the present study also showed a mean volume reduction rate of $68.3 \%$ and a therapeutic success rate of $90.5 \%$, (at the 12 -month followup, or at the last follow-up when the follow-up period was less than 12 months) in a single session, with a mean follow-up period of 18.2 months (range 6-71 months). Our patients had sufficiently large nodules (at least $3 \mathrm{~cm}$ ), and the mean RF active time was 22 minutes, but considering the large thyroid nodule sizes, 22 minutes is actually a short treatment time. If the patient endured the procedure well, the power could be increased to decrease the required treatment time. Consequently, the larger the nodule, the greater the treatment time difference with respect to conventional electrodes.

Even though we conducted a pilot study, and were not able to make a comparison with other published data, we concluded that the longer active tip of the adjustable electrode could create a larger ablation zone more easily and quickly than the conventional fixed-tip electrode. RFA 
with an adjustable electrode may also have the potential to reduce the number of ablation sessions for successful volume reduction in large benign nodules, which usually require more than one RFA session. Moreover, when ablation of a small region is necessary, the shorter tip allows relatively easy adjustment. There were no disadvantages to the adjustable RFA electrode compared to conventional electrodes.

Regarding safety, a previous meta-analysis (19) revealed no major or minor complications (according to the definition in the proposal by the Society of Interventional Radiology) during RFA treatment (20). Jung et al. (17) reported a major complication rate of $1.0 \%(3 / 276)$, and a minor complication rate of $4.0 \%$ (4/276). In another study, Baek et al. (21) demonstrated a major complication rate of $1.4 \%$. In our study, the relatively high minor complication rate $(14.3 \%, 3 / 21)$ was likely due to the small number of subjects. All three patients recovered after manual compression and ointment application.

Several limitations of this study should be addressed. First, due to its retrospective nature, there were irregular follow-up periods, and a lack of selection criteria for treatment of nodules with adjustable electrodes. Second, the patient population was relatively small. Finally, this preliminary study was limited by the lack of comparisons between treatment and control groups, and a lack of data comparisons between fixed and adjustable electrodes. Nevertheless, considering that there have been no published data regarding the usefulness of adjustable active tip electrodes in RFA treatment of thyroid nodules, the present study has significant clinical implications for the treatment of benign symptomatic large thyroid nodules, and may be the basis for larger-scale and longer-term prospective studies.

In conclusion, an adjustable electrode for RFA treatment of benign symptomatic large thyroid nodules can reduce volume effectively and safely. This technique may also have the potential to decrease the procedural treatment time for large nodules, with fewer ablation sessions. We believe that the application of this new electrode in clinical RFA procedures for thyroid nodules will be highly beneficial.

\section{Conflicts of Interest}

The authors have no potential conflicts of interest to disclose.

\section{ORCID iDs}

Jiyeong Lee

https://orcid.org/0000-0001-6586-8802

Jung Hee Shin

https://orcid.org/0000-0001-6435-7357

Soo Yeon Hahn

https://orcid.org/0000-0002-4099-1617

Kowoon Park

https://orcid.org/0000-0001-9386-5772

Ji Soo Choi

https://orcid.org/0000-0003-1361-5269

\section{REFERENCES}

1. Ha EJ, Lim HK, Yoon JH, Baek JH, Do KH, Choi M, et al. Primary imaging test and appropriate biopsy methods for thyroid nodules: guidelines by Korean Society of Radiology and National Evidence-Based Healthcare Collaborating Agency. Korean J Radiol 2018;19:623-631

2. Moon WJ, Baek JH, Jung SL, Kim DW, Kim EK, Kim JY, et al. Ultrasonography and the ultrasound-based management of thyroid nodules: consensus statement and recommendations. Korean J Radiol 2011;12:1-14

3. Bandeira-Echtler E, Bergerhoff K, Richter B. Levothyroxine or minimally invasive therapies for benign thyroid nodules. Cochrane Database Syst Rev 2014;(6):CD004098

4. Mauri G, Cova L, Monaco CG, Sconfienza LM, Corbetta S, Benedini $S$, et al. Benign thyroid nodules treatment using percutaneous laser ablation (PLA) and radiofrequency ablation (RFA). Int J Hyperthermia 2017;33:295-299

5. Kim JH, Baek JH, Lim HK, Ahn HS, Baek SM, Choi YJ, et al. 2017 Thyroid Radiofrequency Ablation Guideline: Korean Society of Thyroid Radiology. Korean J Radiol 2018;19:632655

6. Jeong WK, Baek JH, Rhim H, Kim YS, Kwak MS, Jeong HJ, et al. Radiofrequency ablation of benign thyroid nodules: safety and imaging follow-up in 236 patients. Eur Radiol 2008; 18:1244-1250

7. Spiezia S, Garberoglio R, Milone F, Ramundo V, Caiazzo C, Assanti AP, et al. Thyroid nodules and related symptoms are stably controlled two years after radiofrequency thermal ablation. Thyroid 2009;19:219-225

8. Baek JH, Moon WJ, Kim YS, Lee JH, Lee D. Radiofrequency ablation for the treatment of autonomously functioning thyroid nodules. World J Surg 2009;33:1971-1977

9. Baek JH, Kim YS, Sung JY, Choi H, Lee JH. Locoregional control of metastatic well-differentiated thyroid cancer by ultrasound-guided radiofrequency ablation. AJR $A m \mathrm{~J}$ Roentgenol 2011;197:W331-W336

10. Cha J, Kim YS, Rhim H, Lim HK, Choi D, Lee MW. Radiofrequency ablation using a new type of internally cooled electrode with an adjustable active tip: an experimental study 
in ex vivo bovine and in vivo porcine livers. Eur J Radiol 2011;77:516-521

11. Kim JH, Baek JH, Lim HK, Na DG. Summary of the 2017 thyroid radiofrequency ablation guideline and comparison with the 2012 guideline. Ultrasonography 2019;38:125-134

12. Mauri G, Pacella CM, Papini E, Solbiati L, Goldberg SN, Ahmed M, et al. Image-guided thyroid ablation: proposal for standardization of terminology and reporting criteria. Thyroid 2019;29:611-618

13. Sung JY, Baek JH, Kim KS, Lee D, Yoo H, Kim JK, et al. Single-session treatment of benign cystic thyroid nodules with ethanol versus radiofrequency ablation: a prospective randomized study. Radiology 2013;269:293-300

14. Ha EJ, Baek JH, Lee JH. Moving-shot versus fixed electrode techniques for radiofrequency ablation: comparison in an exvivo bovine liver tissue model. Korean J Radiol 2014;15:836843

15. Baek JH, Ha EJ, Choi YJ, Sung JY, Kim JK, Shong YK. Radiofrequency versus ethanol ablation for treating predominantly cystic thyroid nodules: a randomized clinical trial. Korean J Radiol 2015;16:1332-1340

16. Lim HK, Lee JH, Ha EJ, Sung JY, Kim JK, Baek JH. Radiofrequency ablation of benign non-functioning thyroid nodules: 4-year follow-up results for 111 patients. Eur Radiol
2013;23:1044-1049

17. Jung SL, Baek JH, Lee JH, Shong YK, Sung JY, Kim KS, et al. Efficacy and safety of radiofrequency ablation for benign thyroid nodules: a prospective multicenter study. Korean J Radiol 2018;19:167-174

18. Baek JH, Kim YS, Lee D, Huh JY, Lee JH. Benign predominantly solid thyroid nodules: prospective study of efficacy of sonographically guided radiofrequency ablation versus control condition. AJR Am J Roentgenol 2010;194:1137-1142

19. Ha EJ, Baek JH, Kim KW, Pyo J, Lee JH, Baek SH, et al. Comparative efficacy of radiofrequency and laser ablation for the treatment of benign thyroid nodules: systematic review including traditional pooling and bayesian network metaanalysis. J Clin Endocrinol Metab 2015;100:1903-1911

20. Burke DR, Lewis CA, Cardella JF, Citron SJ, Drooz AT, Haskal ZJ, et al. Quality improvement guidelines for percutaneous transhepatic cholangiography and biliary drainage. J Vasc Interv Radiol 2003;14:S243-S246

21. Baek JH, Lee JH, Sung JY, Bae JI, Kim KT, Sim J, et al. Complications encountered in the treatment of benign thyroid nodules with US-guided radiofrequency ablation: a multicenter study. Radiology 2012;262:335-342 